\title{
The ionizing sources of luminous compact HII regions in the RCW106 and RCW122 clouds
}

\author{
J. M. C. Grave ${ }^{1,3}$, M. S. N. Kumar ${ }^{2,3}$, D. K. Ojha ${ }^{4}$, G. D. C. Teixeira ${ }^{3}$, and G. Pace ${ }^{3}$ \\ ${ }^{1}$ FCNET, Universidade Lusófona do Porto, rua Augusto Rosa 24, 4000-098 Porto, Portugal \\ e-mail: nanda@astro.up.pt \\ 2137 rua Ruben A, 10 Esq, 4150-762 Porto, Portugal \\ 3 Centro de Astrofísica da Universidade do Porto, rua das estrelas, 4150-762 Porto, Portugal \\ 4 Tata Institute for Fundamental Research, Homi Bhabha road, 400005 Mumbai, India
}

Received 17 February 2013 / Accepted 20 January 2014

\begin{abstract}
Context. Given the rarity of young O star candidates, compact HII regions embedded in dense molecular cores continue to serve as potential sites for peering into the details of high-mass star formation.

Aims. We uncover the ionizing sources of the most luminous and compact HII regions embedded in the RCW106 and RCW122 giant molecular clouds, known to be relatively nearby $(2-4 \mathrm{kpc})$ and isolated, thus providing an opportunity to examine spatial scales of a few hundred to a thousand AU in size.

Methods. High spatial resolution $\left(0.3^{\prime \prime}\right)$, mid-infrared spectra $(R=350)$, including the fine structure lines [ArIII] and $[\mathrm{NeII}]$, were obtained for four luminous compact HII regions embedded inside the dense cores within the RCW106 and RCW122 molecular cloud complexes. At this resolution, these targets reveal point-like sources surrounded by the nebulosity of different morphologies, thereby uncovering details at spatial dimensions of $<1000 \mathrm{AU}$. The point-like sources display [ArIII] and [NeII] lines - the ratios of which are used to estimate the effective temperature of the embedded sources.

Results. The derived temperatures are indicative of mid-late $\mathrm{O}$ type objects for all the sources with [ArIII] emission. Previously known characteristics of these targets from the literature, including evidence of disk or accretion, suggest that the identified sources may grow more to become early-type $\mathrm{O}$ stars by the end of the star formation process.
\end{abstract}

Key words. stars: formation - HII regions - infrared: stars

\section{Introduction}

Massive stars play a determining role in shaping the structure and evolution of the Galaxy; however, it seems almost surprising that our understanding of their formation and early evolution, in particular, has barely begun. Forming inside cocoons of dense gas and dust which are not easily probed at short wavelengths, the most well studied early manifestation of a young massive star is the appearance of a compact or ultracompact (UC) HII region, usually detected at radio wavelengths. However, the high column densities associated with these objects prevent us from observing the accreting object directly at this critical early stage, at least until the extinction falls below a few magnitudes in the $K$ band. Furthermore, OB stars are known to form in clusters or multiple systems, which when associated with the large distances where they are usually found, makes it difficult to probe them as isolated entities.

Until recently, only a few objects that can be called O-type young stars have been observationally identified in UCHII regions (Watson \& Hanson 1997; Martín-Hernández et al. 2003), and a large number of the so-called "high mass protostellar candidates" are known to either host accreting B-type stars (Grave \& Kumar 2009), or, are doubted to be clusters. Thus, strong efforts are being made to remove contaminants and to identify a usable sample of massive young stellar objects for followup observations in order to examine the detailed mechanism of high-mass star formation (Mottram et al. 2007). After the signatures of accretion are uncovered in luminous far-infrared targets, establishing the proper content and temperature of the embedded population is crucial for identifying whether the accreting object is indeed an O-type star. Uncovering the embedded star content is possible with high spatial resolution observations (e.g. Feldt et al. 2003), provided the distance to the target is well known and preferably resolved. Only then can pointed observations be undertaken at similar spatial resolutions to evaluate the temperature or mass associated with the candidate high-mass stars.

In this context, the technical evolution in mid-infrared (MIR) observations has provided a useful tool for peering into obscured regions, with a spatial resolution of a few hundred to a thousand AU for targets located closer than $4-5 \mathrm{kpc}$. A number of finestructure lines produced in the ionized regions surrounding massive stars can be observed at these wavelengths, and their analysis can be used to try to uncover the properties of ionizing stars. This method is analogous to what is done with forbidden lines in the optical for more evolved stars. However, they are undetectable in these embedded regions. In some cases, the properties of the ionizing star have been derived using helium and hydrogen lines, which are detected in the near infrared (Hanson et al. 2002). The line intensities and their ratios reflect the ionization status of the elements in UC HII regions. Detecting even these near-infrared lines is quite challenging in $\mathrm{O}$ star-forming regions that are highly extincted and obscured. But they lend themselves to being probed at wavelengths longwards of a few microns where emission from elements such as argon and neon are found. The ionization potentials to form $\mathrm{Ne}^{+}$and $\mathrm{Ar}^{2+}$ are 21.56 and $27.63 \mathrm{eV}$, respectively. 
These are higher values than are required for $\mathrm{H}^{+}$or $\mathrm{He}^{+}$, so the line ratios should be more sensitive to the characteristics of ionizing objects with early spectral types. However, when performing this type of analysis, one should also take the influence of the nebular contamination into account and, especially, the influence of neighbouring ionizing stars on the line intensities, before arriving at any conclusions regarding the nature of an individual massive young star. For example, fine-structure lines have previously been observed in the MIR N-band (Fujiyoshi et al. 1998, 2001) and the far-infrared (FIR; Okada et al. 2010), in an effort to uncover the properties of the ionizing sources in high-mass star-forming regions. However, the observed sources were the combined output of compact clusters, and the results did not lead to a unique interpretation of the nature of the ionizing sources in those clusters.

As such, one factor that can significantly increase the reliability of the results obtained with this method is the spatial resolution with which the observations are done. The diffractionlimited observations with 8-m class telescopes in the MIR band, resulting in a resolution of $\sim 0.3^{\prime \prime}$, allows us to probe the UC HII regions, sampling typical dimensions from a few hundred up to a thousand $\mathrm{AU}$ for targets located within 2-4 kpc of the Sun.

In this work, we present such observations for four highluminosity sources $\left(>10^{4} L_{\odot}\right)$, which are located in nearby (2-4 kpc) star-forming complexes, RCW106 and RCW122, taking advantage of the diffraction limit achieved at the Very Large Telescope (VLT) from the European Southern Observatory (ESO).

\section{The sample}

The targets RCW106 and RCW122, located at distances of $3.6 \pm 0.6 \mathrm{kpc}$ (Lockman 1979) and $2.7 \pm 0.5 \mathrm{kpc}$ (Russeil 2003), respective, were identified as HII regions by their $\mathrm{H}-\alpha$ emission (Rodgers et al. 1960) and are known to be associated with giant molecular cloud complexes (e.g. Lo et al. 2009; Mookerjea et al. 2004; Arnal et al. 2008, and references therein). These clouds are associated with some of the densest and most compact cores harbouring very luminous FIR sources and compact HII regions. RCW106-MMS5 and RCW106-MMS68 stand out as the most massive and densest cores found in the entire RCW106 cloud (Mookerjea et al. 2004) and display intense emission from a large number of molecular species (Lo et al. 2009). Both these cores are associated with extraordinarily luminous FIR sources $\left(>10^{5} L_{\odot}\right)$. Similarly, the cores chosen in RCW122 stand out as the most luminous FIR sources $\left(>10^{4} L_{\odot}\right)$, coinciding with compact HII regions and representing O-type stars (Ghosh et al. 1989). The RCW106-MMS5 core coincides with the G333.60.22 compact HII region known to emit Lyman continuum flux density that can only be achieved by a cluster of several late O type stars (Fujiyoshi et al. 2006). Furthermore, the nearinfrared images analysed by the same authors show that the ionizing sources in this region are represented by unresolved pointlike objects at a level of $1^{\prime \prime}$. In Table 1, we summarize the basic details of the chosen sample.

\section{Observations and data reduction}

The observations were carried out in visitor mode with the MIR VISIR camera, mounted on the Cassegrain focus of the $8 \mathrm{~m}$ VLT UT3 during the nights of 2-3 June 2005. Low-resolution $(R=350$ at $10 \mu \mathrm{m}) N$-band spectra and the respective acquisition images were obtained for each of the targets listed
Table 1. Details of the observed sample.

\begin{tabular}{lclcc}
\hline \hline Name & $\begin{array}{c}\text { RA } \\
(\mathrm{J} 2000)\end{array}$ & $\begin{array}{l}\text { Dec } \\
(\mathrm{J} 2000)\end{array}$ & $\begin{array}{c}d \\
(\mathrm{kpc})\end{array}$ & $\begin{array}{c}L \\
\left(10^{4} L_{\odot}\right)\end{array}$ \\
\hline RCW106-MMS5 & $16: 22: 09.6$ & $-50: 06: 00$ & 3.6 & $<90$ \\
RCW106-MMS68 & $16: 20: 11.2$ & $-50: 53: 15.8$ & 3.6 & 25 \\
RCW122_1 & $17: 20: 07.9$ & $-38: 57: 15$ & 2.7 & $<2.4$ \\
RCW122_2 & $17: 20: 06.9$ & $-38: 56: 59$ & 2.7 & $<2.4$ \\
\hline
\end{tabular}

in Table 1. The sky conditions were excellent, and the system yielded diffraction-limited observations at a spatial resolution of $0.3^{\prime \prime}$. The slit length and width used are $32.3^{\prime \prime}$ and $0.75^{\prime \prime}$, respectively. Four spectral settings overlapping by, at least, $15 \%$ were used to cover the full $N$ band (central wavelengths $=8.8$, $9.8,11.4$, and $12.4 \mu \mathrm{m}$ ). The total on-source integration time was two minutes for each spectroscopic setting. To avoid contamination from the extended diffuse emission, judged by the relatively deep acquisition images with eight seconds of integration time, a chopper throw of $10^{\prime \prime}$, and nodding on slit were applied. Early-type stars were observed before and after each target observation to obtain flux calibration (HD 146791, HD 184996, and HD 171759). In the particular case of RCW106-MMS5, the slit orientation was chosen so that it contained the two bright sources simultaneously (IRS1 and IRS2 in Fig. 1).

The VISIR pipeline, IRAF routines, and customized IDL scripts were used for data reduction. We used the pipeline to perform the subtraction of the chopping and nodding pairs in order to remove most of the sky and telescope background. Any residual background contribution was removed while extracting the spectra by fitting the background emission at each row in the spectral image and subtracting it. The resulting images were shifted and co-added manually to maximize the resulting signalto-noise ratio and spatial resolution. Wavelength calibration and removal of the curvature in the spectroscopic data were done by tracing sky emission lines. Spectra were extracted in different apertures. In the case of RCW106-MMS5, we used smaller apertures than for the other sources in order to extract the spectra of the two MIR sources (IRS1 and IRS2 in Fig. 1). These sources are separated by a distance of 1 ", both embedded in an extended bright nebula. Thus, the extracted spectra may still carry some contamination. Absolute flux calibration was obtained by normalizing the standard star spectra to VISIR narrowband fluxes. In the MIR ground-based observations, it is quite challenging to obtain an accurate flux calibration. We analysed the variability in the sensitivity of the standard star observations throughout the observing run, which lasted five hours, and we noticed a maximum amplitude that yields an accuracy of roughly $50 \%$. Considering that we only calibrate each science target observation with the adjacent standard star observations, the accuracy for the flux calibration of each object spectrum is $30 \%$.

\section{Results}

In Fig. 1, we display the acquisition images of the four observed targets. It can be seen from these images that several details of the observed MIR peaks enclosed within projected diameters of $5000 \mathrm{AU}$ begin to be resolved. We note that the peaks in the RCW106 sources appear point-like in the nearinfrared adaptive optics imaging data obtained with the NACO instrument on VLT (Kumar 2013). In particular, the source IRS2 marked on the RCW106-MMS5 image is a well identified point source at a spatial resolution of $0.1^{\prime \prime}$ in the near-infrared, likely representing an individual star unlike IRS1 which peaks 
J. M. C. Grave et al.: The ionizing sources of luminous compact HII regions in the RCW106 and RCW122 clouds
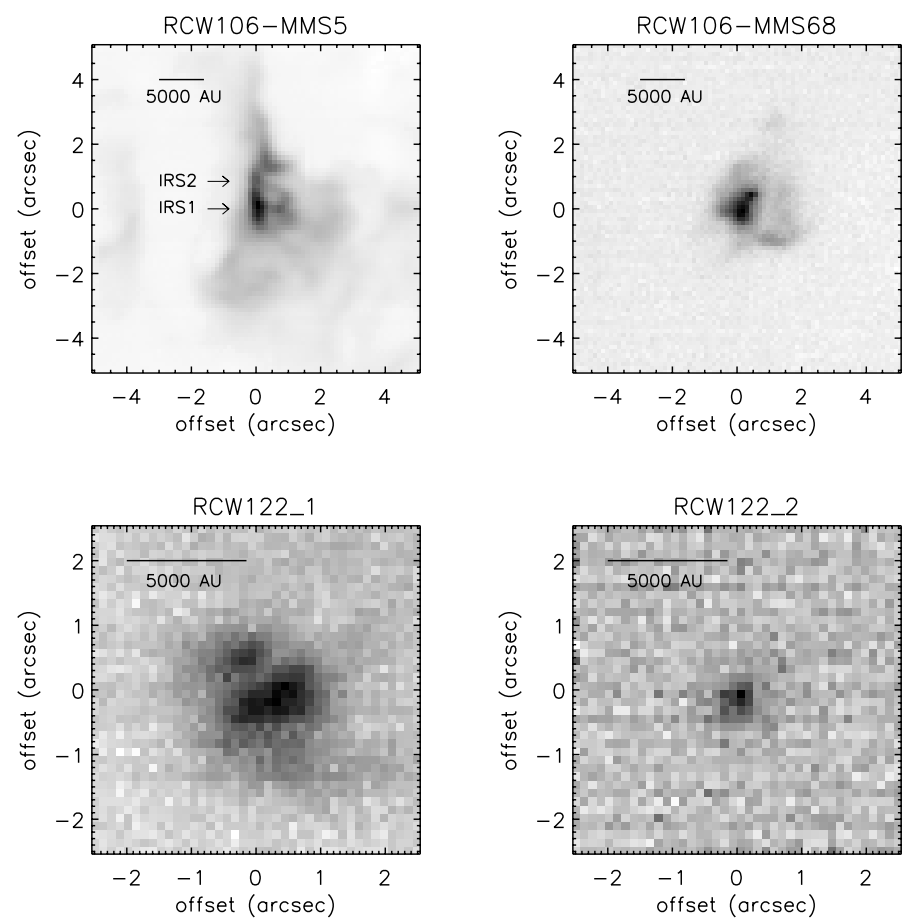

Fig. 1. $N$ band aquisition images of the observed targets for which spectra were obtained. The scale bars indicate a physical size of $5000 \mathrm{AU}$, assuming distances of $3.6 \mathrm{kpc}$ and $2.7 \mathrm{kpc}$ to RCW106 and RCW122, respectively. The offsets marked in the figure are with respect to the positions in Table 1, which correspond to the peak emission in each image, except for RCW 122_1 where it represents the midpoint of the dark lane separating the two emission peaks.

between 8-18 $\mu \mathrm{m}$. At $0.3^{\prime \prime}$ resolution, RCW122_2 and the peak of RCW106-MMS68 appear point-like (Fig. 1) while RCW122_1 reveals a dark lane separated by a compact bipolar nebula. The projected sizes are typically less than $5000 \mathrm{AU}$ (see Fig. 1 for the scale bar). Therefore, the MIR peaks shown in Fig. 1 are in all likelihood isolated objects of high infrared luminosity and not resolved clusters.

In Fig. 2 we present the spectra obtained with VISIR in the $N$ band for the five detected sources in our sample, consisting of four observations. These are wavelength- and flux-calibrated, and the absolute fluxes are shifted by an arbitrary constant for convenience of plotting in a single figure. The [NeII] line at $12.8 \mu \mathrm{m}$ is detected in all the sources, while the [ArIII] at $9 \mu \mathrm{m}$ is also visible in all but RCW122_2. On the other hand, only this source displays the PAH emission feature clearly at $\sim 11.3 \mu \mathrm{m}$. The [SIV] line at $10.5 \mu \mathrm{m}$ typically observed in these type of objects is not detected in these sources, although there is a shallow absorption feature in the spectrum of RCW122_1, which we cannot associate with that line. Recent studies (Furness et al. 2010) have shown that the $[\mathrm{NeII}] /[\mathrm{SIV}]$ ratio is a better diagnostic for obtaining the temperature. In the absence of the [SIV] line, we used the $[\mathrm{ArIII}] /[\mathrm{NeII}]$ diagnostic, taking the uncertainties pointed out by Furness et al. (2010) into account.

Examination of the spectral images shows that for RCW106MMS5, the emission of both lines clearly peak on IRS1 and IRS2 and extends at a low level to fill the region between them. Given that the separation between those sources is about $3600 \mathrm{AU}$, the low-level emission in between the sources may be the combined contribution of both. In RCW106-MMS68, the intensity of the lines is clearly concentrated on the target - a star visible in the near-infrared - with the centroid of the line

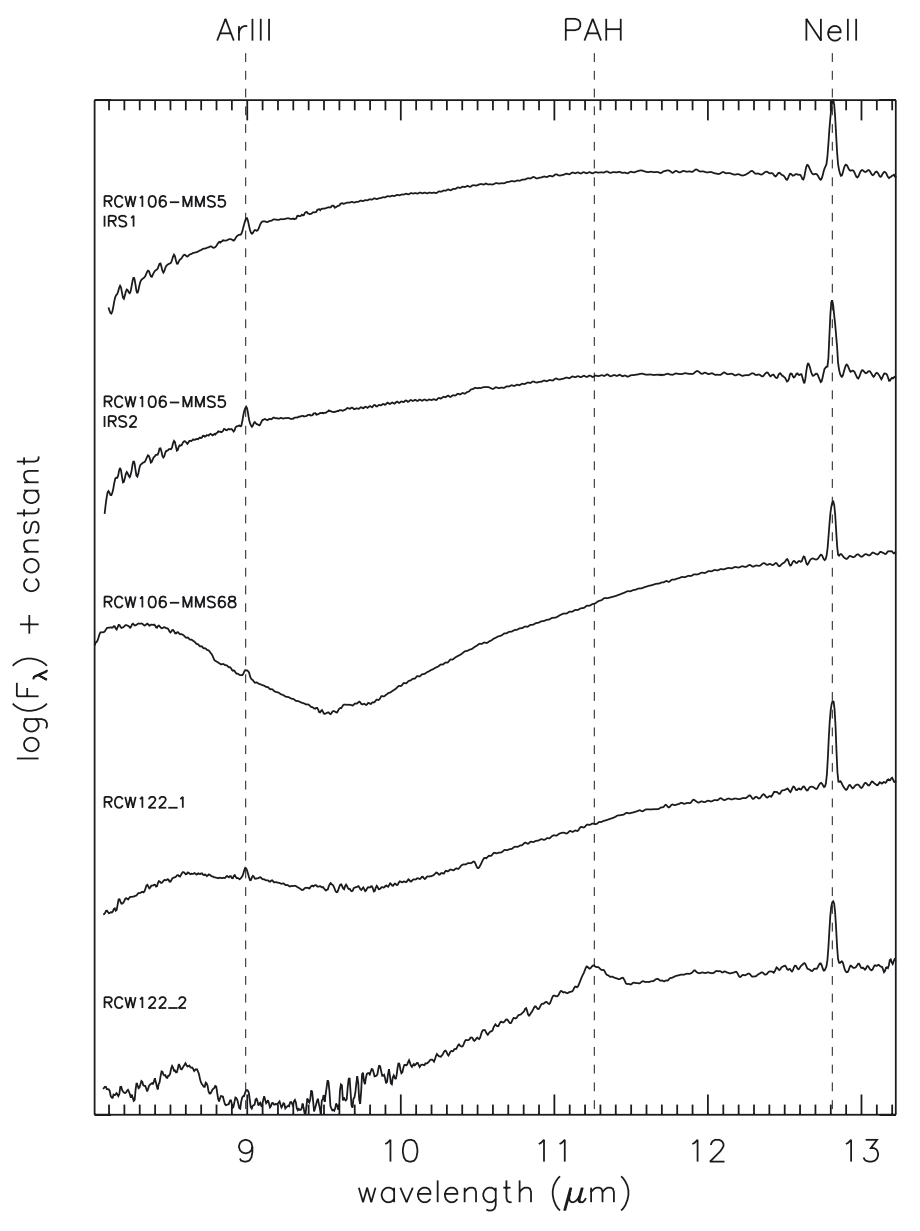

Fig. 2. $N$-band spectra of the observed sources. The dashed lines mark the most prominent spectral features detected.

emission well aligned with the centroid of the continuum strip. In the RCW122 sources, the line emission is also found to be concentrated on the actual targets. However, there are some minor artefacts in the co-added final spectral image, suggesting possible external contamination from beyond the sources.

The line fluxes were calculated for each of the observed lines and corrected for the extinction. Following the work of Furness et al. (2010), we used the extinction-corrected line ratio between the $[\mathrm{ArIII}]$ and $[\mathrm{NeII}]$ lines to obtain the temperature of the observed sources in each region. It was computed using the relation

$\left(T_{\mathrm{eff}} / 10^{3} \mathrm{~K}\right)_{2 Z_{\odot}}=+48.92+12.92 \log \left(\frac{I[\mathrm{ArIII}]}{I[\mathrm{NeII}]}\right)$

derived in the same work for regions with twice the metallicity of the Sun. Given that both RCW106 and RCW122 are located in the fourth Galactic quadrant, with relatively well estimated distances, they should lie closer to the metal-rich Galactic centre, justifying the choice above.

\subsection{Extinction and related uncertainties}

The value of the extinction used to correct the line fluxes has a significant impact on the calculated temperatures. It is difficult to obtain an accurate extinction for these kinds of sources because of numerous uncertainties. The molecular cores hosting these objects are so dense that any attempt to measure extinction using light from background stars is impossible. Measurements using 
Table 2. Observed fluxes, extinctions, and effective temperatures derived using the equation of Furness et al. (2010).

\begin{tabular}{lccccccc}
\hline \hline Source & $\begin{array}{c}F_{\text {ArIII }} \\
\left(\mathrm{erg} \mathrm{s}^{-1} \mathrm{~cm}^{-2}\right)\end{array}$ & $\begin{array}{c}F_{\text {NeII }} \\
\left(\mathrm{erg} \mathrm{s}^{-1} \mathrm{~cm}^{-2}\right)\end{array}$ & $\begin{array}{c}A_{V}(\mathrm{low})^{a} \\
\mathrm{mag}\end{array}$ & $\begin{array}{c}T \text { (low) } \\
10^{3} \mathrm{~K}\end{array}$ & $\begin{array}{c}A_{V} \text { (high) } \\
\text { mag }\end{array}$ & $\begin{array}{c}T \text { (high) } \\
10^{3} \mathrm{~K}\end{array}$ & $\begin{array}{c}\text { SpT } \\
\text { adopted }^{b}\end{array}$ \\
\hline RCW106-MMS5 IRS1 & $2.6 \mathrm{E}-11$ & $1.8 \mathrm{E}-10$ & 16 & $41 \pm 7$ & 119 & $59 \pm 8$ & O5 \\
RCW106-MMS5 IRS2 & $2.5 \mathrm{E}-11$ & $9.6 \mathrm{E}-11$ & 16 & $44 \pm 7$ & 119 & $62 \pm 9$ & O4 \\
RCW106-MMS68 & $2.1 \mathrm{E}-12$ & $1.3 \mathrm{E}-11$ & 13 & $26 \pm 10$ & 108 & $43 \pm 7$ & O9 \\
RCW122_1 & $5.9 \mathrm{E}-14$ & $3.4 \mathrm{E}-12$ & 20 & $29 \pm 10$ & 79 & $40 \pm 8$ & O8.5 \\
RCW122_2 & $\ldots$ & $7.1 \mathrm{E}-13$ & $\ldots$ & $\ldots$ & $\ldots$ & $\ldots$ & $\ldots$ \\
\hline
\end{tabular}

Notes. The assumed uncertainty for the fluxes listed was 30\%. ${ }^{(a)}$ Extinction estimates used for RCW106-MMS5, RCWMMS-68, and RCW122 are from Fujiyoshi et al. (2006), Bik et al. (2006), and Arnal et al. (2008) respectively. ${ }^{(b)}$ See discussion section for the reasoning.

(sub)mm dust continuum emission to calculate column densities often lead to high values of extinction. However, the uncertainties about the dust properties and the degree to which the core may present a clumpy structure may result in large variations of the extinction within a narrow spatial range. Such variations should be averaged out by the large beam of the (sub)mm observations. Therefore, this must be taken into account when using the submm derived extinction values for the analysis of objects with sizes of only a few thousand AU, such as the ones studied in this work. Only RCW122 is associated with a resolved cluster, where an infrared analysis can lead to an average extinction, but it has not been done so far.

Strong controversy about measuring extinction at MIR wavelengths, which affects the lines and the continuum emission to different extents (e.g. Jiang et al. 2006, and references therein). Working within these limitations and without ignoring any measurements, we have adopted both the lowest and highest values of extinction and tabulated the associated temperatures in Table 2. The higher values in Col. 6 of this table are obtained using the uniform dataset from the ATLASGAL survey (Schuller et al. 2009). The targets are assumed to be located at the centre of the dense cores traced by $870 \mu \mathrm{m}$ dust continuum observations. The peak flux of the associated cores obtained from the ATLASGAL database was used to compute the column densities. Following Draine (2003), the extinction for each source was calculated using the relation $A_{V}=N_{\mathrm{H} 2} \times 6.71 \times 10^{-22}$. Considering the dense and dusty cores associated with the sources analysed in this work, we have adopted $R_{V}=5.5$, since it corresponds to an extinction law that is more representative of the denser regions of molecular clouds, as shown for other, similar regions (Chapman et al. 2009).

\section{Discussion and conclusions}

As shown in the previous section, the $0.3^{\prime \prime}$ diffraction limit of the VLT MIR observations provides a spatial resolution that represents physical scales below $1000 \mathrm{AU}$ for all four observed targets, uncovering several details of the MIR peaks. The temperatures estimated by analysing nebular lines from these peaks are indicative of hot $\mathrm{O}$ type stars. In fact, Hanson et al. (2002) point out that for those objects with both NIR and MIR data, the temperature estimated from MIR nebular line analysis are systematically lower than photospheric temperatures. The issue here is whether more than one O-type star can contribute to the measurements presented here on a physical scale of $1000 \mathrm{AU}$. Theoretical arguments propose that some level of multiplicity is expected either due to $N$ body interactions (Bonnell et al. 2003; Bonnell \& Bate 2005) or to the fragmentation of massive disks (Kratter \& Matzner 2006; Kratter et al. 2010), which results in multiple objects. However, such mechanisms do not produce equal mass objects on such small physical scales, which can dominate the ionizing radiation within the MIR peaks. In fact, equally dominant sources are expected to be found at larger separations (e.g. Krumholz et al. 2009) of greater than a thousand AU, which would be resolved as independent MIR peaks through our observations, as is the case for RCW106MMS5. A study of binarity in massive stars (Sana et al. 2012), focussing on evolved stages, also supports this conjecture. Therefore, the results presented in this work describe the nature of the most dominant ionizing source, typically representing O-type stars, even though a low level of contamination from multiple sources is expected.

The temperature estimates of spatially resolved MIR peaks in this work, such as sources IRS 1 and IRS 2 associated with RCW106MMS5, may represent objects of different masses at different evolutionary stages. An exact description of the true nature and physical conditions around the embedded high-mass star is beyond the reach of our observations because the photosphere is not traced. Even so, the wealth of information on these luminous targets from previous studies allows us to speculate about some characteristics. If these sources were to represent zero-age-main-sequence stars, our results could be extrapolated to spectral types (Vacca et al. 1996). In contrast, if they are younger objects, similar to bloated pre-main sequence objects (e.g. Ochsendorf et al. 2011), representing accreting stages, it would imply that they will become hotter and more massive when star formation is complete. Given that the chosen sources are all embedded inside compact HII regions with association of dense molecular gas, we can safely rule out that they represent evolved objects. Infrared and radio observations on angular scales of an arcsecond are available for the sources in the RCW106 cloud but not for the RCW122 sources. Each of the targets are discussed in detail below.

RCW106-MMS5: the targets studied here coincide with the core of the UCHII region associated with the MMS5 core. A H90 $\alpha$ radio recombination line study of this compact region, surrounding targets IRS1 and IRS2, displays double-peaked profiles, indicating a complex kinematic structure (Fujiyoshi et al. 2006). These authors modelled this region to be associated with an outflow originating in a compact object that represents a cluster of $\mathrm{O}$ type stars. These results, combined with the the small spatial extent of the sources in the MIR clearly excluding the possibility of clusters or multiple systems of ionizing stars and with the information that only two stars (IRS1 and IRS2) are found in this region, imply that the objects are at an early evolutionary stage and, at least one of them is, in all likelihood, are still accreting. The lower limit of temperature in Table 2 suggests that they are similar to O6 main-sequence stars, while the upper limit would place them at the Wolf-Rayet limits. Since these two are the most luminous point-like objects within the 
compact HII region, producing a LyC flux equivalent to 12 O7 type stars, the lower and upper limits both seem unreasonable. However, if we assume that IRS1 and IRS2 are in the range of O3-O5 spectral types, much of the observed data, in particular the bolometric luminosity and the LyC flux, can be coherently explained.

RCW106-MMS68: this source was studied using VLTISAAC spectroscopy by Bik et al. (2006) (object 16164nr3636), which led them to model the CO-band head emission to arise in a disk. They also suggest that the embedded source is of spectral type $\mathrm{O} 9 \mathrm{~V}$ to interpret the origin of the nebular HeI emission. The extinction of 13 visual magnitudes, as adopted by Bik et al. (2006), is clearly an under-estimation of the real value, as stated by the authors, because it was derived from neighbouring, more evolved stars. The presence of the CO band heads and the deep 10-micron silicate absorption visible in the respective spectrum in Fig. 1, indicates a higher degree of obscuration.

Spectro-astrometric analysis of the $\mathrm{Br} \gamma$ line from this object indicated the presence of an expanding HII region (Grave \& Kumar 2007) that traces size scales of a few hundred AU. Considering the analysis of Bik et al. (2006), their estimated spectral type is well within the temperature limits presented in this work. This is clearly an excellent target for follow-up observations at other wavelengths and comparable spatial resolution, to peer into the mechanisms of $\mathrm{O}$ star formation.

RCW122_1 \& RCW122_2: these objects are the two main MIR peaks located at the heart of the largest clump traced by radio and FIR observations. The two objects ( $1 \& 2)$ are separated by $\sim 17^{\prime \prime}$ and are identified as RCW122 A \& B by Ghosh et al. (1989). This region appears as an elongated object with a single peak on the $870 \mu \mathrm{m}$ images obtained through ATLASGAL. The molecular clump clearly hosts an embedded cluster, which is traced by resolved stellar population visible in the 2MASS and Spitzer-IRAC data. RCW122_1 reveals itself to be a spatially extended object, separated by the dark lane representative of an edge-on disk like object. The true driving engine may actually be hidden, and it is possible that our observations trace the scattered light within outflow cavities. Even so, the computed value for the temperature is high, suggesting a late O-type object. We think that RCW122_1 is of the spectral type O8.5, because it consistently explains the lack of an [ArIII] line in the RCW122_2 source, combined with the total bolometric luminosity observed for these two sources (Ghosh et al. 1989).

In summary, the high spatial and low spectral resolution MIR observations of the luminous embedded targets in the nearby high-mass star-forming regions from our sample have led us to identify their ionizing sources. The large uncertainties in extinction measurements for these objects, and the lack of detection of [SIV] lines, prohibit an accurate description of the targets. Even so, the temperature analysis presented here, together with other diagnostics from the literature, clearly suggests that these are mid-late O-type objects, three of which may evolve to become early $\mathrm{O}$ stars.

Acknowledgements. The authors are grateful to the referee, Dr. Lex Kaper, for constructive and useful comments. This work was carried out under the auspices of a European Union Marie-Curie IRSES grant (230483). This work is based on the data obtained through the ESO VLT proposal id 075.C-0833A.1.

\section{References}

Arnal, E. M., Duronea, N. U., \& Testori, J. C. 2008, A\&A, 486, 807 Bik, A., Kaper, L., \& Waters, L. B. F. M. 2006, A\&A, 455, 561 Bonnell, I. A., \& Bate, M. R. 2005, MNRAS, 362, 915

Bonnell, I. A., Bate, M. R., \& Vine, S. G. 2003, MNRAS, 343, 413

Chapman, N. L., Mundy, L. G., Lai, S.-P., \& Evans, N. J., II 2009, ApJ, 690, 496 Draine, B. T. 2003, ARA\&A, 41, 241

Feldt, M., Puga, E., Lenzen, R., et al. 2003, ApJ, 599, L91

Fujiyoshi, T., Smith, C. H., Moore, T. J. T., et al. 1998, MNRAS, 296, 225 Fujiyoshi, T., Smith, C. H., Wright, C. M., et al. 2001, MNRAS, 327, 233

Fujiyoshi, T., Smith, C. H., Caswell, J. L., et al. 2006, MNRAS, 368, 1843 Furness, J. P., Crowther, P. A., Morris, P. W., et al. 2010, MNRAS, 403, 1433

Ghosh, S. K., Iyengar, K. V. K., Rengarajan, T. N., et al. 1989, ApJS, 69, 233

Grave, J. M. C., \& Kumar, M. S. N. 2007, A\&A, 462, 37

Grave, J. M. C., \& Kumar, M. S. N. 2009, A\&A, 498, 147

Hanson, M. M., Luhman, K. L., \& Rieke, G. H. 2002, ApJS, 138, 35

Jiang, B. W., Gao, J., Omont, A., Schuller, F., \& Simon, G. 2006, A\&A, 446, 551

Kratter, K. M., \& Matzner, C. D. 2006, MNRAS, 373, 1563

Kratter, K. M., Matzner, C. D., Krumholz, M. R., \& Klein, R. I. 2010 ApJ, 708, 1585

Krumholz, M. R., Klein, R. I., Mckee, C. F., Offner, S. S. R., \& Cunningham, A. J. 2009, Science, 323, 754

Kumar, M. S. N. 2013, A\&A, 558, A119

Lo, N., Cunningham, M. R., Jones, P. A., et al. 2009, MNRAS, 395, 1021 Lockman, F. J. 1979, ApJ, 232, 761

Martín-Hernández, N. L., Bik, A., Kaper, L., Tielens, A. G. G. M., \& Hanson, M. M. 2003, A\&A, 405, 175

Mookerjea, B., Kramer, C., Nielbock, M., \& Nyman, L.-Å. 2004, A\&A, 426, 119

Mottram, J. C., Hoare, M. G., Lumsden, S. L., et al. 2007, A\&A, 476, 1019

Ochsendorf, B. B., Ellerbroek, L. E., Chini, R. et al. 2011, A\&A, 536, L1

Okada, Y., Kawada, M., Murakami, N., et al. 2010, A\&A, 514, A13

Rodgers, A. W., Campbell, C. T., \& Whiteoak, J. B. 1960, MNRAS, 121, 103

Russeil, D. 2003, A\&A, 397, 133

Sana, H., de Mink, S. E., de Koter, A., et al. 2012, Science, 337, 444

Schuller, F., Menten, K., Contreras, Y., et al. 2009, A\&A, 504, 415

Vacca, W. D., Garmany, C. D., \& Shull, J. M. 1996, ApJ, 460, 914

Watson, A. M., \& Hanson, M. M. 1997, ApJ, 490, L165 